\title{
Instead of Quantitative Analysis in Improving the Technical Sports
}

\author{
Benlakehal Mansour University of Mostaganem \\ Alouane Rachid, Art and Physical Activities and Sport Sciences and Practice \\ laboratory. University of Alger 3,
}

Alouane-staps@hotmail.fr

\section{Introduction}

Our goal is to answer the initial question on the role of biomechanical analysis of technique in shot put in improving performance in the Algerian athlete. We will try to see if there is a significant difference between the performance achieved before biomechanical analysis and the analysis followed by a training program that focuses on the shortcomings and misconduct. We will also note the performances respectively. Much more, we will compare the second execution (post-program) that the model takes on the champion of Algeria discipline. Athletic performance is a multi-factorial phenomenon. It is the subject of numerous investigations across different scientific fields. If the disciplines of the humanities and life sciences allow the coach to take a look at the meaning attributed to the athlete's motor and biological dispositions that enable it to realize, biomechanics enables them to understand the human locomotion, to detect the most efficient gestures and possibly correct errors.

A better understanding of sporting gesture is one of the factors in improving its performance. Biomechanics, "science that studies the internal and external forces acting on the human body and the effects produced by them (Hay, 1986, p.216), allows the coach to analyze the gesture, namely make technical corrections to better performance. Robin (1973, p.145), speaks of the biomechanical examination, and he says "it is essential to carefully consider the whole of a gesture about what can be, a priori, assign a technical failure, which often comes from a weakness in one link of the chain joints and muscles". Regarding to Geoffrey (1975, p.11), he describes the knowledge of the biomechanics of essential means of distinguishing the important accessory, correct the incorrect.

Through an experiment, which is to compare the performance of two pitches thrown by a beginner athlete to that of a champion considered an execution model, the first launch of the beginner will be conducted to assess the technical level latter and draw fouls to avoid.

The second launch will be made after workouts that will aim to work and correction of errors in the first run. The method used for analysis and evaluation of three throws is biomechanics. That said we ask the following question: biomechanical analysis does improve the performance significantly in the Algerian launcher?

Biomechanics has two lines of research: quantitative analysis and modeling. Our research is part of the first axis; it is to a biomechanical analysis of OBRIEN technique in shot put and its importance in improving performance among Algerian athletes.

The quantitative analysis of human movement is mostly made of cinematic and / or dynamic tools. By the early 1870s, the work of Marey and Demeny, it became possible to objectively measure the performance gestures using dynamo-graphic and / or chrono-photographic analyzes (Marey, 1873). The sports movement is difficult to study because of its complexity and sometimes changing properties and system of the area in which it operates. The dynamic analysis tools (force platform, plantar pressure sensors ...) not to define the segmental contributions involved in the topic, the characteristics of sports movements and environmental constraints of the activity are the three-dimensional kinematic analysis tool most often used to reconstruct the gestures of the subjects. Nowadays, the most efficient systems use at least two planar projections of motion to reconstruct three-dimensional (Yeadon and Challis, 1994; See Fig 1).

The kinematic analysis systems can be classified into two categories depending on the use or not of markers on the subject studied. Systems using multiple markers are (infrared, ultrasonic, etc...). Markers, placed at each joint, to identify the position of the segments in an image of the subject. The use of markers on the subject shows that these analysis systems are not used in competition. However, the majority of the most 
successful gesture made by top athletes is observable only during these tests. To allow analysis of these gestures without using cooperation of the subject.

The Modeling thus allows developing new strategies and gestures to predict their influences on performance. The appropriation of a model requires the sports biomechanics to consider the representations that the athlete uses in his activity and observable parameters by the coach. The use of common paradigms in robotics and the study of systems consisting of poly-articulated sound are now possible to offer much more faithful in representations of athletes. Models that represent the subject in its entirety promote understanding of the influence of a strategy gestural performance. The dispersion of performance between elite athletes is very low; these performances usually provide more information in line with expectations of the coach. Already used in many scientific fields, modeling is a particularly effective means of investigation in cases where the design comes from a rigorous method.

The shot put is a discipline that belongs to the family of athletics. This is an acyclic movement and also "an activity where the action is carried out in an isolated form, closed in on itself, the motor form is characterized by a phase structure in three parts" (Beyer, 1987, p. 91).

In terms of technique O'Brien, it is broken down according to some authors, in three parts and in other four parts. Hopf (1977), which was decomposed into four gesture parts called: preparation, sliding, throwing and finally catching up.

With regard to Hinz (1983), there are three parts called successively, start shaving, acceleration and finally catch up.

Then Fleuridas (1990), who joined the previous author on the number of phases, but he uses different names. He describes successively, preparatory, implementation, and at the end finish with a final phase.

For our part, we distinguish, for ease of study the four following phases (Technique of O'Brien):
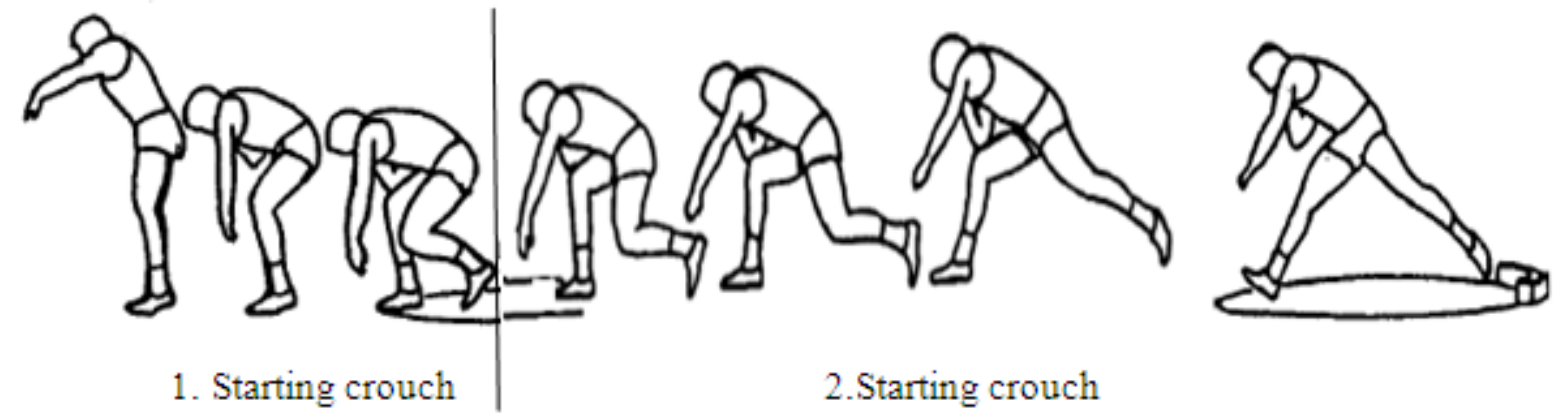

\section{Starting crouch}

2.Starting crouch
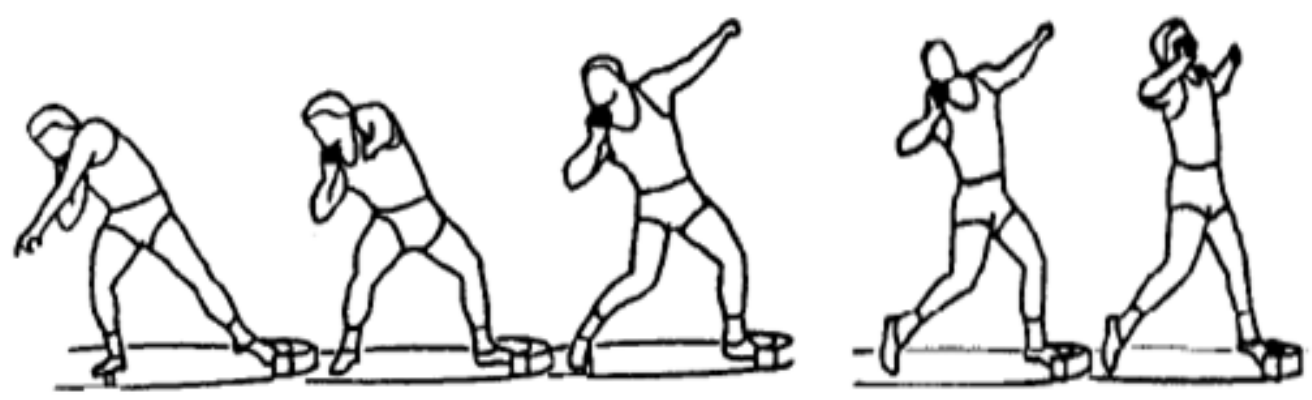

3.The power position
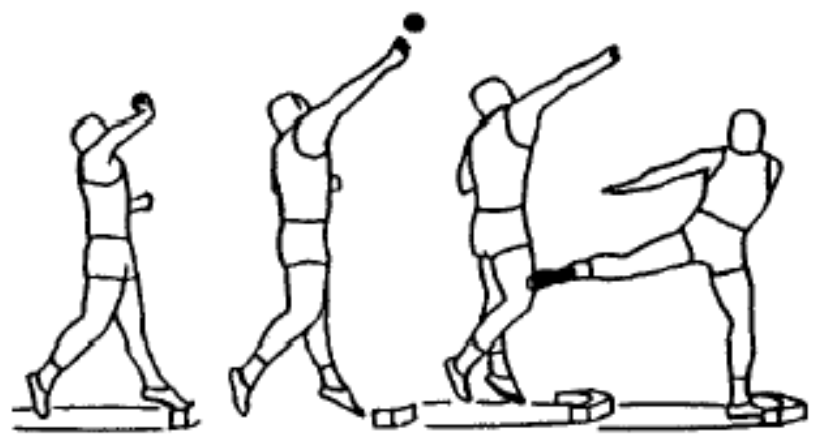

4.The delivery position 
Fig.1- The O'Brien technique divided into 4 phases (Hopf, 1977).

Experimental plan:

II. Materials and Method

Throws registration:
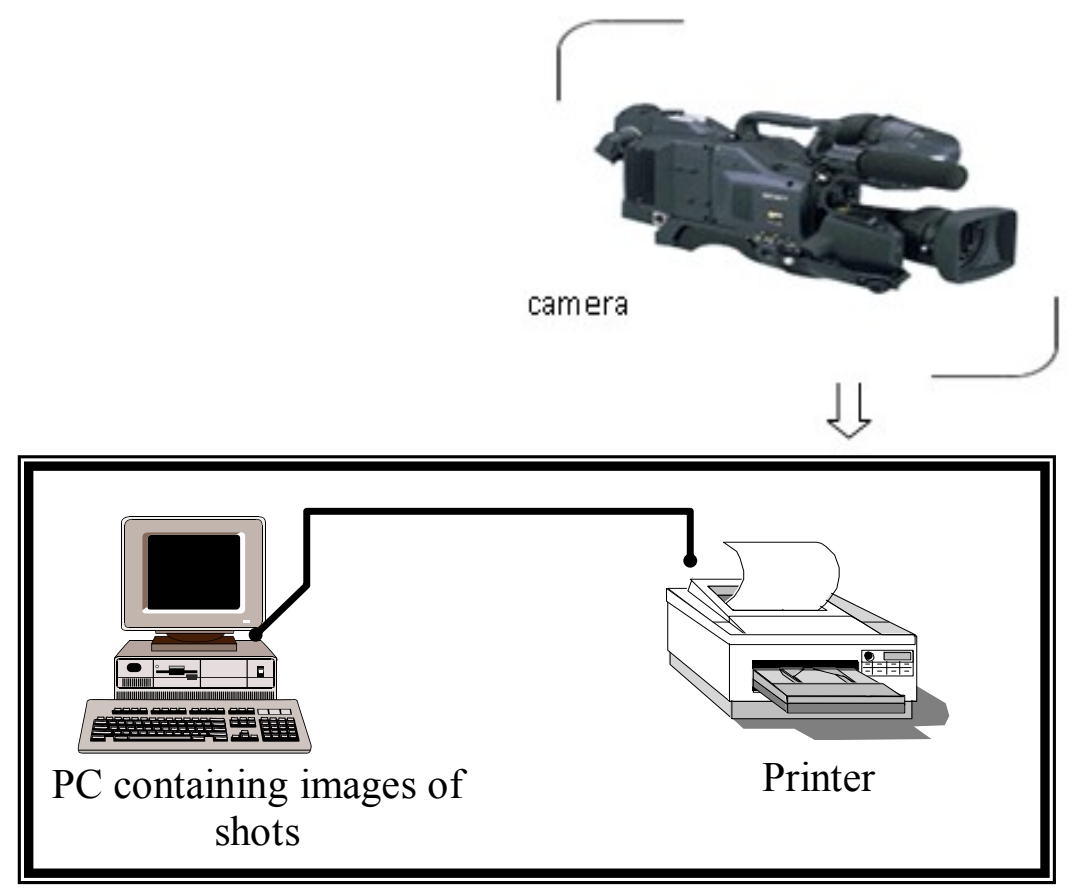

Printing images of the (04) technical phases

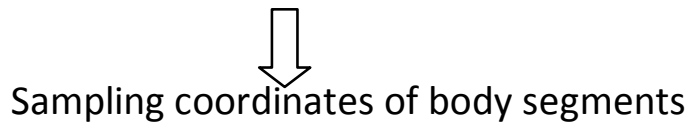

on images printed throws

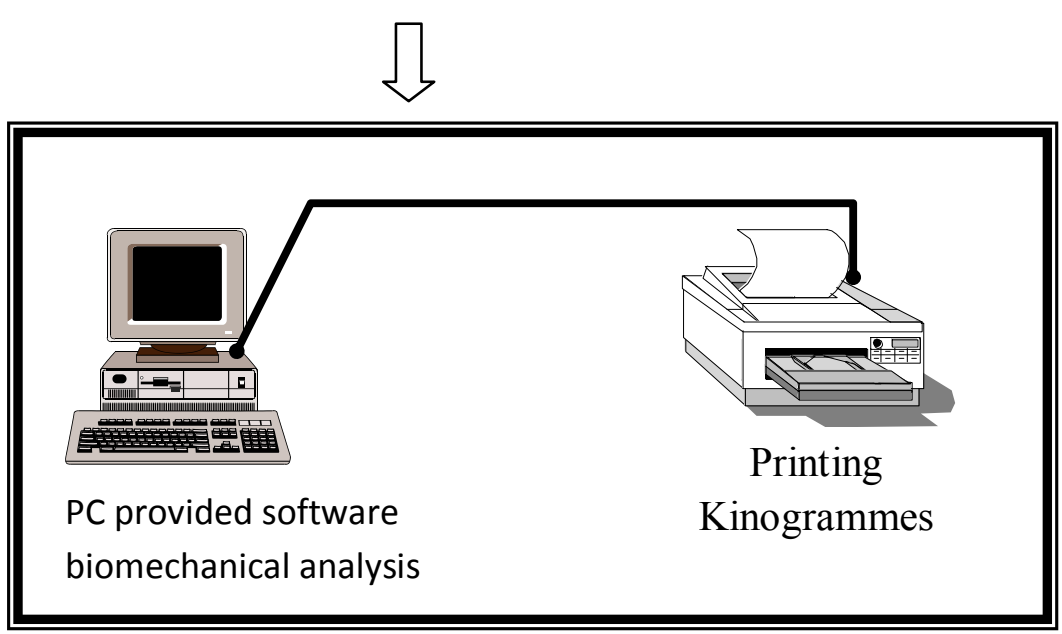

Introduction coordinates and obtaining Kinogrammes of different achievements 
In light of these technical points that characterize the correct execution of the shot put, we believe that the information provided by biomechanical analysis will be very useful from the second phase where technical difficulties are more important.

\section{The course of the experiment:}

The subject performs the shots from a single position. He is wearing shorts. The joints are marked with fluorescent elastic bands. The performance of each throw is recorded. Athletes perform three tests; we will choose the sequence of better performance.

\section{Error factors:}

We tried to reproduce the same material conditions for recording shots, but errors investment material was still presented.

During collection of data (Easting and Northing) images, we found it difficult to locate some joints, which probably led to miscalculations and possibly measure.

Due to the quality of the film, we have encountered difficulties to have the same phases (with the same beginning and the same end) for all shots (thanks to the freeze frame). This led, no doubt, errors in the calculation of the average trajectory of C.G.G.

\section{The Kinogrammes of three shots:}

\section{Results}

Following our experiment, we obtained the following Kinogrammes and their performances: Those of the athlete:

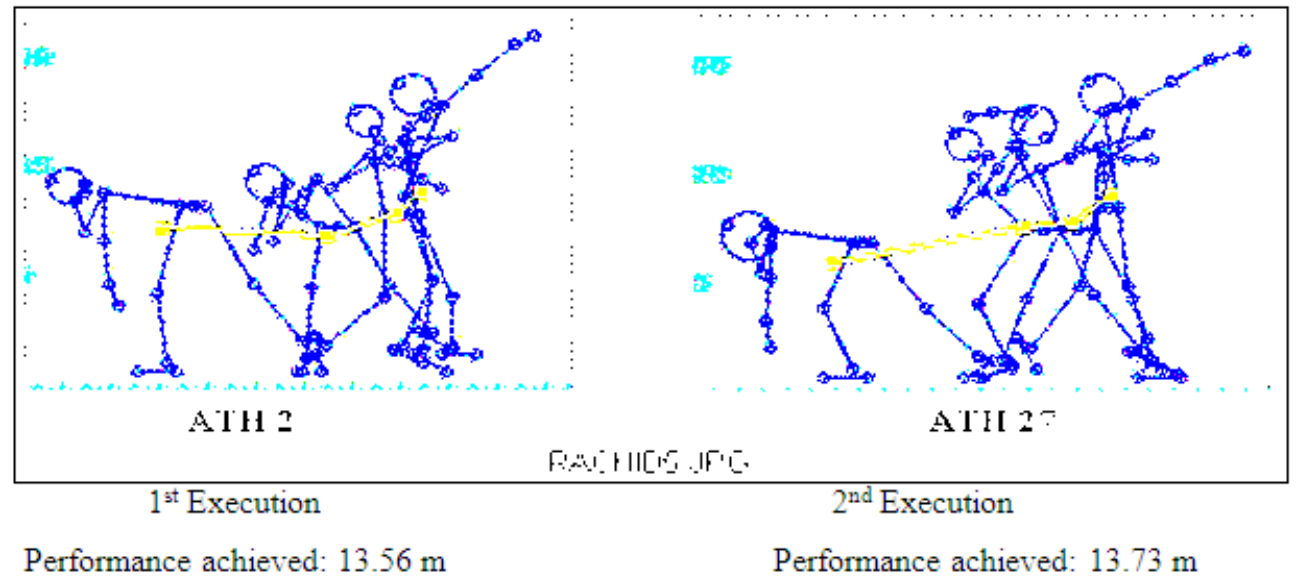

Those of the model:

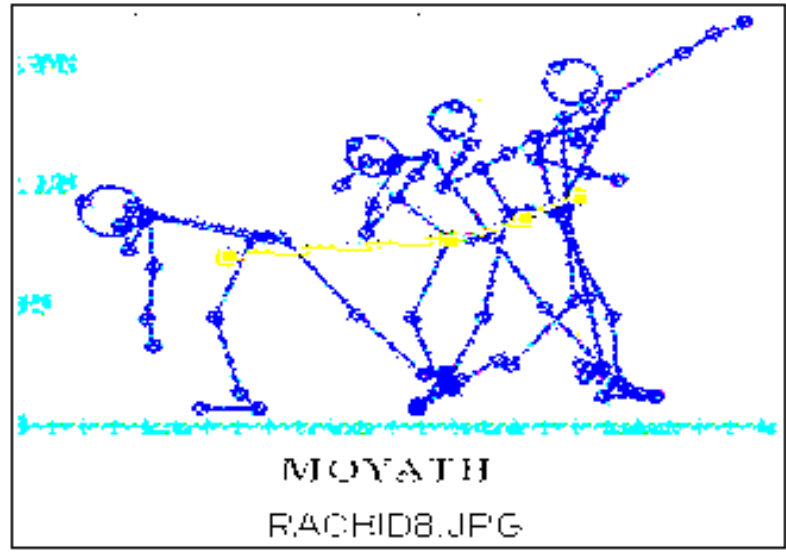

Performance achieved: $13.53 \mathrm{~m}$

Description of trajectories of the general center of gravity (CGG):

Those of the Model: Following the sticks that mark the path of CGG, we note that it rises gradually from the beginning until the end of the throw. It's an upward trajectory (gradual increase), without sharp, smooth and 
without breaking change, it has evolved vertically $0.35 \mathrm{~m}$ and horizontally $1.20 \mathrm{~m}$ and he made a throw of $15.53 \mathrm{~m}$.

Those of the Athlete:

First performance: Compared to the trajectory of the model, the execution of the athlete was jerky. Also, we notice a pronounced slump in the starting glide and a sharp rise in the power phase. The vertical displacement was $0.20 \mathrm{~m}$ and the recovery was $1.35 \mathrm{~m}$. Performance achieved was $13.56 \mathrm{~m}$.

Second performance: compared to the trajectory of G.D.G model, we see overall approximation, it appears at the starting glide where the slump noted during the first execution disappears and it makes way for a qualified climb still mainly caused by sudden premature recovery of the trunk. It has evolved vertically $0.45 \mathrm{~m}$ and horizontally $1.50 \mathrm{~m}$. The performance achieved was $13.73 \mathrm{~m}$.

Technical errors in the first execution compared to the model:

1. During starting crouch:

* A lack of deflection of the right leg.

* The elbow of the right arm is not enough away from the body.

* Shoulders and pelvis are not horizontal.

All of its anomalies gave an investment center of gravity relatively high in relation to his own body and also that of the model.

\section{During the glide:}

* The bust is too low during this phase. Left arm was too relaxed.

* The right leg which is brought under the body was stretched, and then it should be bent slightly, to give a lower center of gravity between both femora (compared to that of the model).

\section{During the power position:}

* The weight of the body rests on a too bent right leg.

* A premature detachment of chin's weight.

\section{During the delivery position:}

Upon the delivery of the put, the legs should be fully extended, which is not the case because the legs still slightly bent.

Following a training program or sessions focused on work and correction of technical errors highlighted previously, the beginner athlete was straightened his path CGG particularly at the stage of start and the power, therefore this was an opportunity to improve performance of $0.17 \mathrm{~m}$. What is significant in this discipline or changes in the results are long and very hard to observe.

\section{Conclusion}

The purpose of this experiment was to provide a technical of shot put for a novice athlete approaching the illustrated form the champion of the discipline. Certainly it is not to imitate but to respect the general principles of execution. As we have seen biomechanical analysis allows us to place a precise technical shortcomings and weaknesses of the athlete, it illuminates the coach on the way it was executed on technical movement. During technical learning sessions, it must be attached in parallel with sessions improving physical or motor skills.

It is clear that the mechanics of human movement approach continues to evolve both in the evaluation and the optimization of the gesture. Technological development of assessment tools and optimizing the movement promotes understanding and the discovery of the most powerful gestures. Coaches concerned about the performance of its athletes cannot just simply a qualitative empirical look at the movement. He has an interest in seeking the biomechanics insofar as their close cooperation contributes to the development of tools tailored to the coach.

\section{References}

[1]. BAYER E. « Dictionnaire des sciences du sport » Ed. Verlag, R.F.A, 1992.

[2]. FLEURIDAS \& Co «Traité d'athlétisme »: Vol 2, Ed. Vigot, Paris, 1990.

[3]. HAY.J G : « Biomécaniques des techniques sportives », Ed Vigot, Paris, 1980.

[4]. Kendall M.G. «La construction des modèles et les problèmes qui s’y rattachent ». Revue de statistique appliquée, paris, 1969.

[5]. Marey E.J « La machine animale ». Réédition de l'œuvre originale en 1993, ed EPS. 1873.

[6]. Yeadon M.R., Challis J.H. « The future of performance related sports biomechanics research ». Journal of Sport Sciences, 1994. 